\title{
Property-driven development of privileged macrocyclic scaffolds using heterocycles
}

\author{
George J. Saunders and Andrei K. Yudin* \\ Davenport Research Laboratories, Department of Chemistry, University of Toronto, Toronto, Ontario, Canada, M5S 3H6. \\ E-mail: andrei.yudin@utoronto.ca
}

KEYWORDS: cyclic peptides, permeability, heterocycles, conformation, peptidomimetics

\begin{abstract}
Passive membrane permeability is a fundamental challenge in the development of bioactive macrocycles. To achieve this objective, chemists have resorted to various strategies, the most common of which is deployment of $N$-methylated amino acids and/or D-amino acids. Here we investigate the effect of heterocyclic grafts on the passive membrane permeability of macrocycles and report the structural consequences of iterative amino acid replacement by azole rings. Through stepwise substitution of amino acid residues for heterocycles, we show that lipophilicity and PAMPA permeability of a macrocycle can be vastly improved. Overall, changes in permeability do not scale linearly as more heterocycles are incorporated, underscoring the subtleties of conformationproperty relationships in this class of molecule. NMR analysis and molecular dynamics simulations provide insights into the structural consequences of the added heterocycles and foreshadow the emergence of privileged macrocyclic scaffolds for drug discovery.
\end{abstract}

\section{Introduction}

Cyclic peptides have emerged as important scaffolds in drug discovery due to their large polar surface area and their capacity to interact with difficult targets that are not accessible to small molecules. ${ }^{1-4}$ However, the relatively large polar surface areas of cyclic peptides commonly confer poor membrane permeability, which significantly hampers their development towards intracellular targets. ${ }^{5-8}$ The capacity for a peptide macrocycle to cross membranes greatly depends on its $3 \mathrm{D}$ conformation in solution, which can be controlled by backbone modification. ${ }^{9,10}$ The major contributing factors that are known to be important include shielding of polar groups by hydrophobic side chains, as well as achieving balance between open and closed orientations upon transition between the aqueous environment and the lipophilic interior of the membrane. ${ }^{11-15}$ This is the key mechanism by which cyclosporin A, a macrocyclic undecapeptide, exhibits unusually high permeability $\left(-\log P_{e}=6.6\right)$ despite its many polar groups and amide linkages. ${ }^{16}$ To mimic this, modifications such as introduction of D-amino acids, peptoid motifs and lipophilic groups have been explored (Figure 1a). ${ }^{17-19}$ Seminal work by Lokey has shown the importance of amide $\mathrm{N}$ methylation, which removes a hydrogen bonding donor, adds extra lipophilicity and can organise the backbone into conformations that are highly permeable (e.g 1-NMe-3, Fig 1a). This can also be achieved with selectivity towards solvent exposed amides within a macrocycle. ${ }^{18,20}$ Recently, Fairlie and co-workers have shown that $N$ - or $\mathrm{C} \alpha$ - methylation can lead to increased permeability by connecting or extending hydrophobic patches in macrocyclic scaffolds. ${ }^{21}$ In a study on cyclic hexapeptides, Kessler determined that there is no linear relationship between the number of $N$-methyl groups added to the backbone and the permeability of the macrocycle. ${ }^{22,23}$

The properties of peptide macrocycles are defined by the stereochemistry, allylic strain, and amide $E / Z$ isomerism
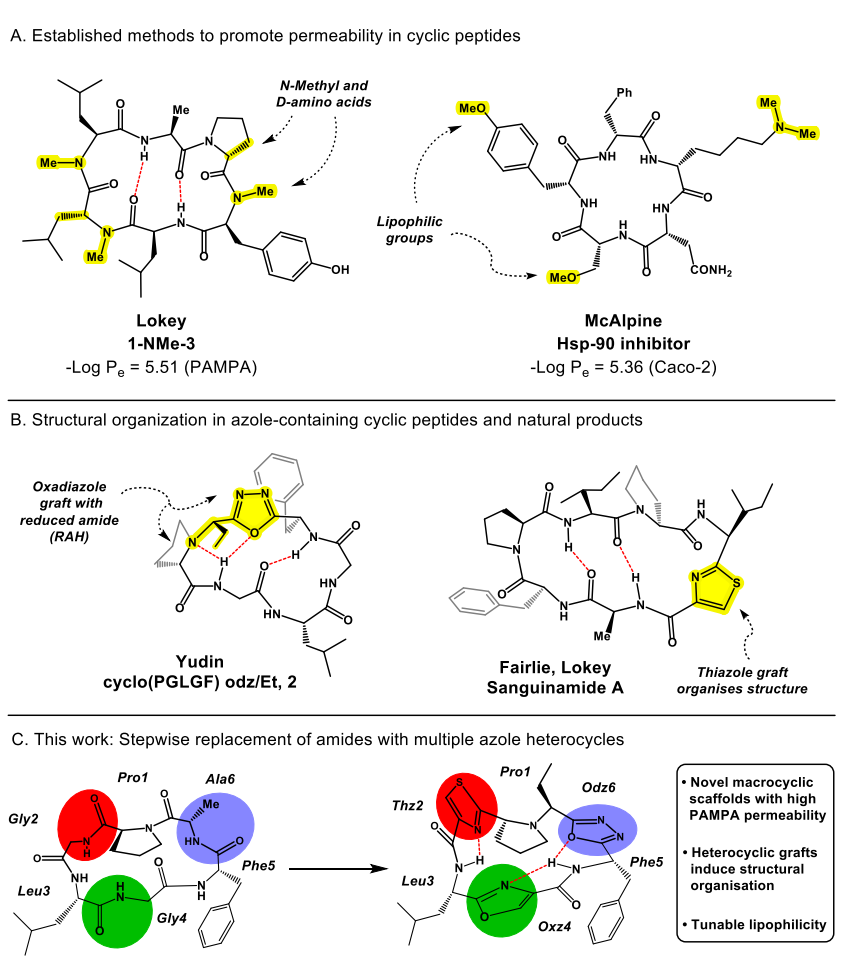

Figure 1: A) Current state-of-the-art methods to improve permeability in peptide macrocycles. B) Examples of structural organization in peptide macrocycles by incorporation of a single azole heterocycle. C) Schematic overview of the work presented in this study. 
inherent to $\alpha$-amino acid residues. ${ }^{9}$ The interplay between these factors is manifested in allowable dihedral angles that describe a peptide bond. While amino acid $N$-methylation or introduction of D-amino acids can lead to an improvement in cellular permeability, we sought more drastic measures to perturb the local geometric preferences of amino acid residues. The intrinsic planarity of aromatic heterocycles was appealing to us, and we hypothesized that the macrocyclic backbone could be forced to adopt distinctly different local conformational states, which might result in previously unappreciated outcomes when considered in aggregate. Site-selective grafting of medicinally relevant motifs should enable the discovery of other scaffolds which are amenable to structure-activity studies akin to small molecules.

The emergence of library-based methods such as SICLOPPS and RaPID have revolutionized the discovery of biologically active macrocycles withe high selectivity and specificity towards challenging intracellular targets. ${ }^{24-26}$ However, the physicochemical properties of the leads generated by library methods can be highly variable, especially if they contain all- $L$ amino acids or polar side chains. ${ }^{27,28}$ Given the struggles associated with low cellular permeability of large peptide molecules, a privileged scaffold must engender a reasonably permeable starting point for further structure/activity studies. In the present paper we demonstrate the application of this strategy to 18-membered rings, an important and diverse class of peptide macrocycles. ${ }^{29}$

\section{Results and Discussion}

Previously, we reported the synthesis of 1,3,4-oxadiazolebased cyclic peptides generated from an efficient macrocyclization between a linear peptide, an aldehyde and $N$-(isocyamino)triphenylphosphorane (PINC). ${ }^{30}$ The oxadiazole ring and reduced amide (RAH) play important roles in the structural organization of these scaffolds across a range of ring sizes, such as in 18-membered $\mathbf{2}$, which adopts a $\beta$-turn-like motif around the oxadiazole and a Type-II $\beta$-turn around the peptide region (Figure 1b). ${ }^{31}$ Similarly, a thiazole ring within the macrocyclic natural product Sanguinamide A stabilizes two $\beta$-turns yet the homodetic control containing Ala in its place exhibits conformational flexibility. ${ }^{32,33}$ These findings, amongst others, highlight the ability of heterocycles to promote structural organisation within cyclic peptides (Figure 1b). ${ }^{34-36}$ In the case of Sanguinamide $\mathrm{A}$, the homodetic control shows similar permeability to the natural product. Furthermore, the macrocyclic natural product Patellamide $\mathrm{C}$ contains several heterocyclic linkages and displays excellent permeability. ${ }^{37}$ These reports, while interesting, do not address the question of how heterocycles tend to influence macrocycle conformation and permeability. In fact, the Sanguinamide A case exemplifies the "reverse" logic because the heterocycle in a natural product that possesses baseline cellular permeability is being replaced by an amide to substantiate the point that amide bonds can result in undesirable properties. In this paper our goal was to understand how gradual depeptidization of a peptide backbone with heterocyclic grafts effects their structure and permeability and whether this strategy could generate a new class of permeable macrocyclic scaffolds (Figure 1c).

Our investigations began by searching for a macrocyclic system and a set of heterocycles to incorporate. We decided to use 18-membered cyclo(PGLGFA) 1 for several reasons. It is known to be difficult to achieve permeation in an all- $L$ peptide backbone, ${ }^{28}$ and $\mathbf{1}$ is known to display poor permeability (Table 1). Additionally, $\mathbf{1}$ exhibits a mixture of conformations at room temperature on the NMR timescale, offering the possibility to investigate changes in structural organization as a result of heterocyclic grafting. ${ }^{30}$ Motivated by previous work with oxazole and thiazole-based systems alongside our own research with 1,3,4-oxadiazole-based RAH, we chose these as the heterocycles to incorporate into the backbone. Dipeptide building blocks containing oxazole and thiazole in replacement for a single amino acid can be made in gram-scale quantities for use in solid phase peptide synthesis (SPPS) from commercially available starting materials and serve as trans-amide bond isosteres. ${ }^{38,39}$ We used this approach to produce linear peptides that contained one or two heterocyclic grafts using conventional $\mathrm{Fmoc} /{ }^{t} \mathrm{Bu}$ SPPS with 2-chlorotrityl resin. Subsequently, eleven macrocyclic counterparts of homodetic peptide 1 were produced, each containing one, two or three different grafts within the backbone. This allowed us to compare between macrocycles with grafts in different positions around the ring and with different numbers of heterocycles in the backbone whilst maintaining the 18-membered ring size. The linear peptides underwent head-totail macrocyclization with the coupling agent DEPBT, producing amide-linked macrocycles $\mathbf{1}, \mathbf{3}, \mathbf{4}$ and $\mathbf{5}$. In addition, we utilized our PINC-mediated macrocyclization with propionaldehyde to form macrocycles 2 and 6-11, which contained an ethyllinked oxadiazole RAH in the backbone. A key consideration for oxadiazole-containing macrocycles is the stereochemistry of the ethyl linker that is generated during the cyclization step. The stereochemistry was determined to be $(R)-\mathbf{6}$ and $(S)-\mathbf{8}$ after

Table 1: Scope of macrocycles containing multiple heterocyclic grafts.

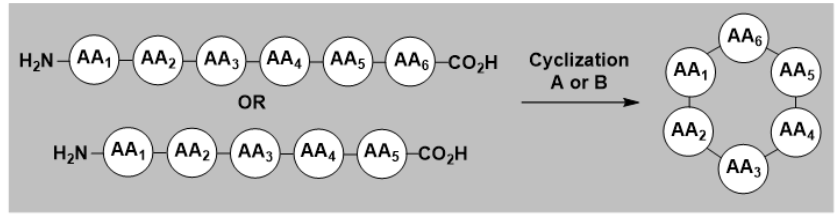

\begin{tabular}{|c|c|c|c|c|c|c|c|c|c|}
\hline \multirow[t]{2}{*}{ Entry } & \multicolumn{6}{|c|}{ Sequence } & \multirow[t]{2}{*}{ No. of Het } & \multirow[t]{2}{*}{-Log $P_{\text {app }}$} & \multirow[t]{2}{*}{$T_{\text {Ret }}$} \\
\hline & $\mathbf{A A}_{1}$ & $\mathrm{AA}_{2}$ & $\mathrm{AA}_{3}$ & $\mathbf{A A}_{4}$ & $\mathrm{AA}_{5}$ & $\mathbf{A A}_{6}$ & & & \\
\hline 1 & Pro & Gly & Leu & Gly & Phe & Ala & 0 & $>8$ & 6.14 \\
\hline 2 & Pro & Gly & Leu & Gly & Phe & Odz & 1 & 7.75 & 6.90 \\
\hline 3 & Pro & Gly & Leu & Oxz & Phe & Ala & 1 & 7.19 & 7.64 \\
\hline 4 & Pro & Thz & Leu & Gly & Phe & Ala & 1 & 7.35 & 7.33 \\
\hline 5 & Pro & Thz & Leu & $O x z$ & Phe & Ala & 2 & 5.84 & 9.47 \\
\hline 6 & Pro & Gly & Leu & Oxz & Phe & Odz & 2 & 7.62 & 7.76 \\
\hline 7 & Pro & Thz & Leu & Gly & Phe & Odz & 2 & 6.29 & 8.84 \\
\hline 8 & Pro & Thz & Leu & Oxz & Phe & Odz & 3 & 5.72 & 10.76 \\
\hline 9 & Pro & Thz & Leu & $O x z$ & Tyr & Odz & 3 & 6.07 & 8.89 \\
\hline 10 & Pro & Thz & Phe & $0 x z$ & Phe & Odz & 3 & 5.85 & 10.72 \\
\hline 11 & Val & Thz & Leu & $0 x z$ & Phe & Odz & 3 & 6.25 & 12.00 \\
\hline
\end{tabular}

Permeability and physical property data for macrocycles 1-11. Yield over all steps from 2-chlorotrityl chloride resin, conducted on $0.3 \mathrm{mmol}$ scale. Cyclization conditions A: 1.5 equiv. DEPBT, 3.0 equiv. DIPEA, DMF, $5 \mathrm{mM}$, RT, 48 h; Cyclization conditions B: 1.5 eq. propionaldehyde, 1.0 equiv. PINC, $1: 1 \mathrm{DCE} / \mathrm{MeCN}, 25$ $\mathrm{mM}, \mathrm{RT}, 3 \mathrm{~h}$. For full details, see the ESI. -Log $\mathrm{P}_{\text {app }}$ determined using PAMPA with carbamazepine as an internal standard. Retention time determined using LC-MS, gradient $95 \% \mathrm{H}_{2} \mathrm{O} / 5 \% \mathrm{MeCN}$ to $100 \% \mathrm{MeCN}$ over $15 \mathrm{~min}$. 
analysis of NMR-restrained MD simulations for each possible diastereomer (see Supplementary Information).

With a suite of macrocycles containing varied numbers of heterocycles in hand, the physicochemical properties were measured for each compound (Table 1). The passive permeability of each derivative was determined using a parallel artificial membrane permeability assay (PAMPA). The reverse-phase HPLC retention time was also collected with a gradient method under identical conditions. This metric has been shown to relate to permeability in peptide macrocycles, as longer retention times correlate with lower aqueous solubility, lower polarity and greater lipophilicity. ${ }^{40-42}$ Each of the compounds containing one or more heterocycle showed improved permeability compared to the homodetic control 1. In general, as the number of heterocycles in the backbone increases, the permeability also increases. Interestingly, the same trend was seen for retention times, which went up as more heterocyclic grafts were added to the backbone in place of polar amide bonds. This confirmed our hypothesis that heterocycles can increase lipophilicity, either by stabilizing non-polar conformations or by reducing polarity by replacing amide linkages. It was not particularly surprising to note that incorporation of only one heterocycle led to small increases in permeability. Investigations into derivatives of Sanguinamide A showed that the homodetic control containing Ala in place of a thiazole displayed very similar permeability by Caco-2 assay. ${ }^{33}$ Within our study, significant improvements were noted upon introduction of a second heterocycle to the backbone, although this did not hold true for $\mathbf{6}$ bearing an oxazole and oxadiazole RAH component. This lack of predictability in physical properties as more heterocycles are added highlights that it is not trivial to understand the impact of grafting on structure and permeability, which demonstrates the need for a systematic scaffold development. To our delight, cyclo(PThzLOxzF) odz/Et 8, which contains three heterocycles and only two amide bonds within its backbone, displays the highest permeability of the set. To probe the effect of different side chains on the permeability of compound $\mathbf{8}$, we prepared $\mathbf{9}$, 10 and 11. Each side chain position is tolerant to change without large decreases in permeability, although a slight reduction is seen when switching Prol to Vall. This could be due to unmasking of the tertiary amine in $\mathbf{8}$ to a secondary amine in $\mathbf{1 1}$ and suggests that the rigid proline motif is a key residue for the organisation of the structure. Importantly, switching Phe in $\mathbf{8}$ to the polar side chain Tyr in $\mathbf{9}$ did not lead to large changes in permeability, suggesting that polar residues can be tolerated within the backbone. Moreover, the retention time of this compound decreased by almost two minutes, indicating that the aqueous solubility of these compounds can be tuned without great changes in permeability. ${ }^{41,42}$ Given that the compounds bearing three grafts all showed excellent permeability despite changes to their side chains, this framework exemplifies a new class of privileged structure which addresses a key challenge in macrocycle development.

Macrocyclic peptides with more than five amino acids generally fall outside of the rule-of-five. ${ }^{5}$ In fact, the doubly grafted macrocycles 5, 7 and the triply-grafted macrocycles 8-11 are all non-Ro5 compliant. Kihlberg and co-workers have studied the structural motifs which promote high permeability in a macrocyclic natural product library of 20,998 members, $70 \%$ of which fell outside of the Ro5. It was found that tertiary amines and isoxazoles are favourable functional groups, but carbonyl groups are not. ${ }^{43}$ The installation of a tertiary amine by macrocyclization to form the RAH, alongside the replacement of carbonyl groups with similar azole heterocycles to isoxazole, support the permeability improvement observed when heterocycles are added to the backbone. Our grafts in place of amide bonds reduce the amount of HBD in total, although with overall increase in HBA. Considering these compounds show permeability values that are superior to Cyclosporin A, which is considered an excellent standard as an orally available, non-Ro5 compliant macrocyclic peptide drug, it is clear that heterocyclic grafting can have a great impact on the physical properties of peptide macrocycles. ${ }^{44}$

\section{Structural analysis of grafted macrocycles}

In order to understand the structural effects of heterocyclic incorporation into the backbone, each compound was fully characterized using ${ }^{1} \mathrm{H}$ and ${ }^{13} \mathrm{C}$ NMR techniques. Solution structures were obtained from molecular dynamics simulations using NOE-derived interproton distances derived from ROESY spectra (See ESI). Additionally, temperature coefficients were determined from VT-NMR experiments, with coefficients of 4 $\mathrm{ppb} / \mathrm{K}$ or lower indicating that the amide bond was either involved in an intramolecular hydrogen bond (IMHB) or is nonsolvent exposed. ${ }^{45}$ A general observation that we noticed in structures grafted with oxazoles and thiazoles was that the azole ring forces the preceding amide bond to be coplanar in a syn conformation with the heterocycle, to minimize electronic repulsion between the $\mathrm{C}=\mathrm{O}$ group and the azole nitrogen (Figure 2 ). This observation is consistent across structures of the grafted macrocycles in this study and in oxazole and thiazole containing macrocycles in the literature. ${ }^{46}$ However, H-bonds do not always form between the amide NH's and the azole nitrogen in close proximity.

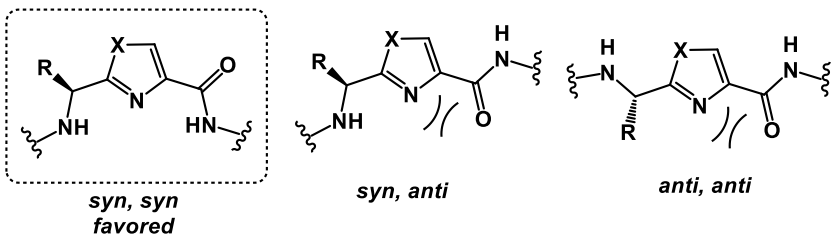

Figure 2: Conformational preferences of dipeptide-based azole heterocycles. $X=O$ or $S$. $R=$ amino acid side chains. ${ }^{46}$

We sought to understand the impact of a single heterocycle on the structure of homodetic sequence cyclo(PGLGFA) 1. Replacement of Gly2 with thiazole in cyclo(PThzLGFA) 4 retained the 7:3 mixture of conformational isomers seen in $\mathbf{1}$, likely as a result of cis/trans isomerization about the proline amide bond (Figure 3, right). However, introduction of an oxazole to Gly4 instead of thiazole to Gly2 led to the adoption of a single set of signals in the ${ }^{1} \mathrm{H}$ NMR spectrum of cyclo(PGLOxzFA) 3. The solution structure of $\mathbf{3}$ displays a very similar backbone to cyclo(PGLGF)odz/Et 2 containing a Type II $\beta$-turn, but in a different position around the macrocycle (Figure 3 , left). This explains their similar permeability values, although 3 exhibits a longer retention time than 2 as a result of more effective shielding of the remaining amides by side chain residues.

It is critical to understand how addition of a second heterocycle to each derivative alters their structure and permeability. Of the three, cyclo(PGLOxzF) odz/Et 6 bearing oxazole and oxadiazole grafts in the backbone displayed lower permeability 


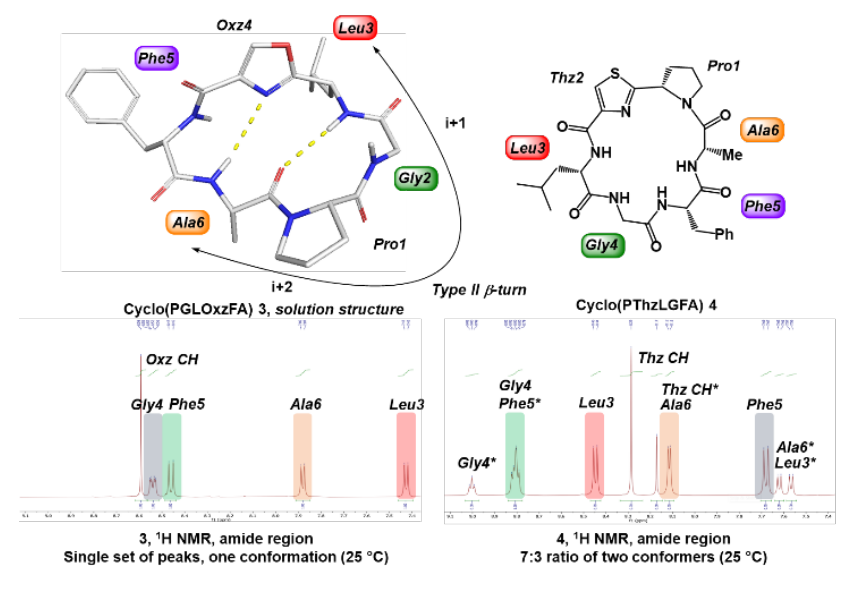

Figure 3: NMR and structural comparison of singly grafted macrocycles 3 and 4 .

and retention time compared to the other doubly grafted macrocycles. In $\mathbf{2}$, the amide bond between Leu3 and Gly4 is solvent exposed. Upon oxazole incorporation to this position in $\mathbf{6}$, there was complete structural reorganisation and interruption of the $\beta$-turn seen in 2 (Figure 4, top right). In fact, two of the three remaining amide bonds in $\mathbf{6}$ are now solvent exposed, alongside the Gly NH that engages in a hydrogen bond with the RAH nitrogen. This explains the dramatic reduction in permeability compared to the other doubly grafted macrocycles, despite an overall increase in retention time compared to $\mathbf{1}$ and $\mathbf{2}$. This result is important because it confirms that the conformation of the macrocycle is the key characteristic in determining permeability within these systems. The other Gly residue in $\mathbf{2}$ is the $\mathrm{i}+2$ position supporting a Type II $\beta$-turn, so grafting in this position was expected to rearrange the structure significantly. The ${ }^{1} \mathrm{H}$
NMR spectra of 7, bearing a thiazole in this position, displayed a 5:1 ratio of conformational isomers with significant overlap between $\mathrm{NH}$ amide protons, indicative of a flexible backbone (Figure 4, bottom). EXSY cross-peaks were observed in the ROESY spectrum, confirming that these two species were in rapid exchange on the NMR timescale (Figure 4, bottom right). This prevented the generation of a solution structure as it was not possible to unambiguously characterize the major rotamer from overlapping peaks in the ROESY spectrum. Alongside the fact that conformational flexibility can be important for permeability, ${ }^{47}$ the observation of low VT NMR coefficients for Gly4 and Leu3 in the major rotamer (Gly4, -2.8 ppb/K, Leu3, -3.7 $\mathrm{ppb} / \mathrm{K}$ ) suggests that the conformations of 7 contain several non-solvent exposed amide bonds and likely enables reasonable permeation compared to $\mathbf{6}$.

Amongst the highest permeability compounds in the set is cyclo(PThzLOxzFA) 5, which contains both oxazole and thiazole grafts replacing both Gly2 and Gly4 in 1. A single set of sharp peaks was observed in the ${ }^{1} \mathrm{H}$ NMR spectrum, indicative of a rigid structure. The backbone conformation of $\mathbf{5}$ displays a bifurcated hydrogen bond between the Leu3 $\mathrm{NH}$ and the oxazole and thiazole nitrogen atoms (Figure 5). Since the permeability of this compound is very similar to that of triply grafted macrocycle 8 , we were motivated to understand the similarities between the two structures. When the oxadiazole RAH was incorporated in place of Ala6 in cyclo(PThzLOxzF) odz/Et 8, a similar backbone conformation to $\mathbf{5}$ is retained, with direct transposition of the Phe5/Ala6 amide with the oxadiazole ring, and replacement of the $\mathrm{Ala} 6 \mathrm{C}=\mathrm{O}$ with the ethyl linker (Figure 5). The similarities in the conformation of these compounds explain why they exhibit similar physical properties. It can be determined that the oxadiazole graft is not critical for high permeability, given that macrocycles with or without the RAH exhibit
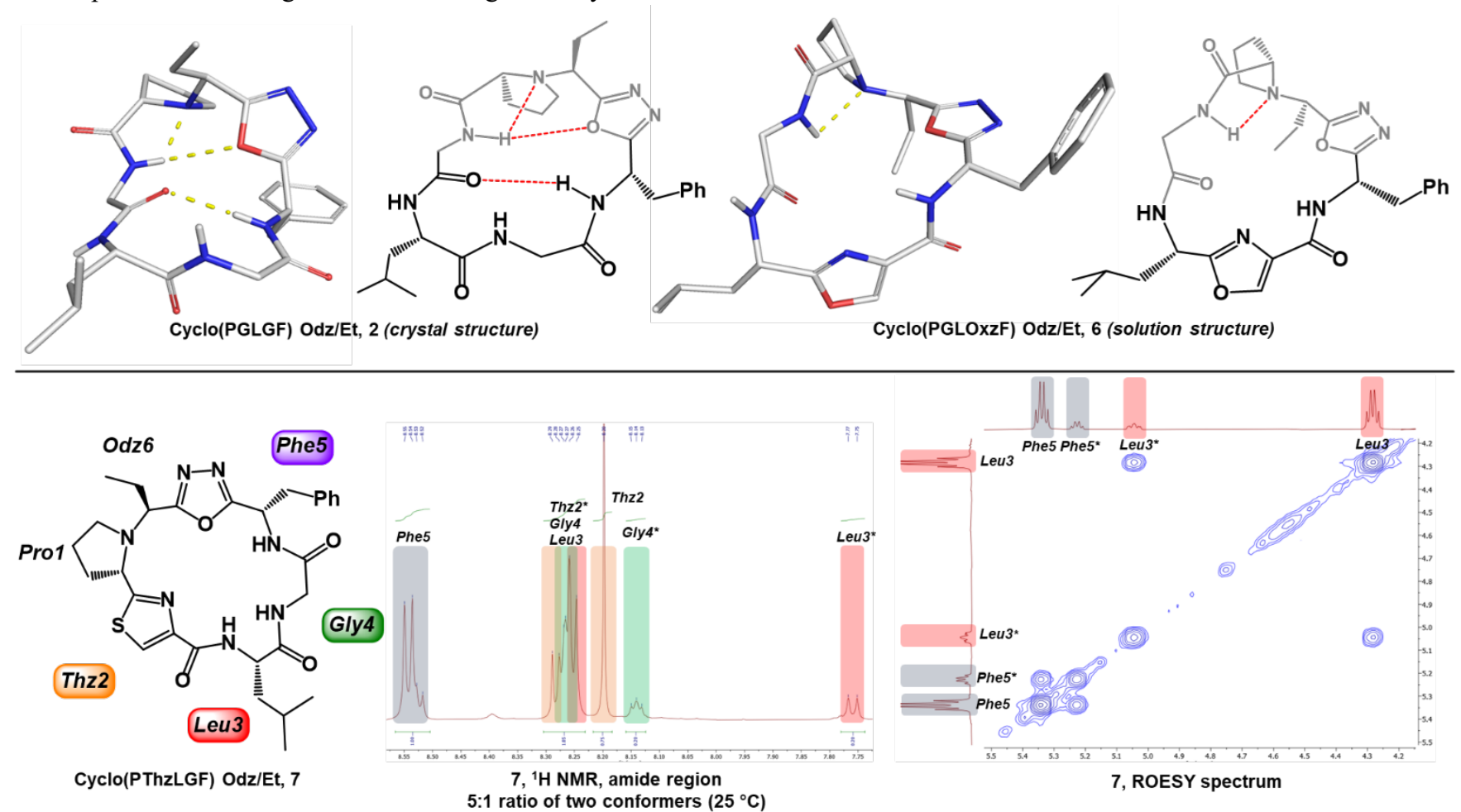

Figure 4: Structural implications of adding two heterocycles to different positions of oxadiazole based macrocycle 2. 


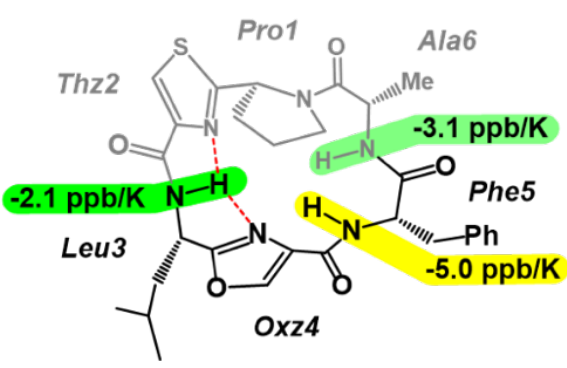

5 (DMSO)

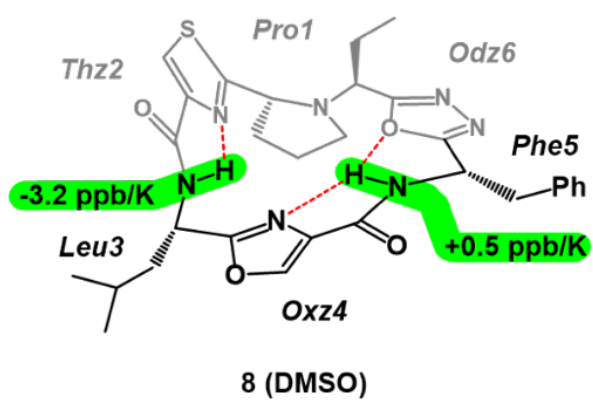

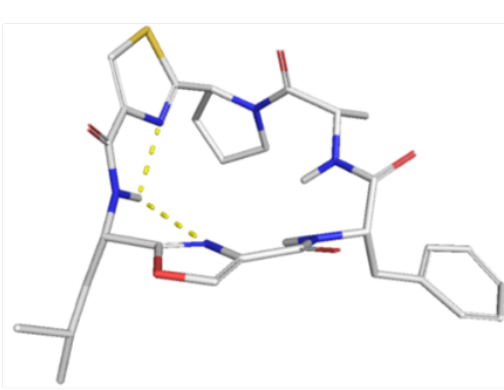
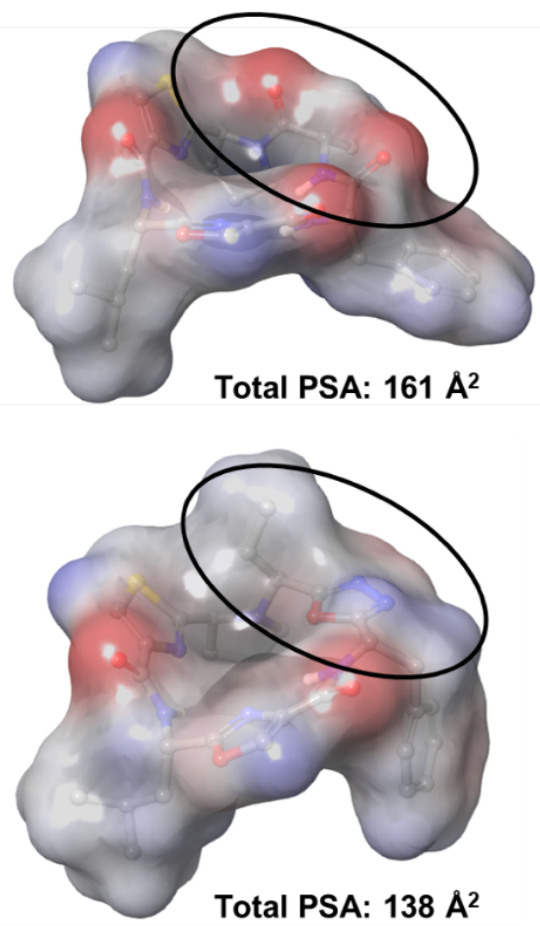

Figure 5: Structural characterization of NMR restrained solution structures of 5 and 8 (left) and polar surface areas of 5 and 8 computed using Maestro (right).

similar permeability. Rather, the combination of oxazole and thiazole adjacent to each other stabilizes a conformation where the remaining amide NH's point into the ring and are not exposed to solvent. The importance of the oxadiazole graft is apparent when comparing the temperature coefficients for each of the amides in $\mathbf{5}$ and $\mathbf{8}$. Whilst Leu3 and Ala6 are well shielded from solvent, Phe5 is not (Leu3, $-2.1 \mathrm{ppb} / \mathrm{K}$, Phe5, $-5.0 \mathrm{ppb} / \mathrm{K}$, Ala6, $-3.1 \mathrm{ppb} / \mathrm{K})$. Placement of the oxadiazole adjacent to Phe5 in 8 engages this amide in a strong bifurcated hydrogen bond, confirmed by very low temperature coefficients (Phe5, +0.5 $\mathrm{ppb} / \mathrm{K}$, Leu3, -3.2 ppb/K). Further effects were observed when analysing the polar surface areas of $\mathbf{5}$ and $\mathbf{8}$ respectively. The importance of connected hydrophobic surfaces on passive membrane permeability has been explored by Fairlie and coworkers. $^{21}$ Replacement of the polar Ala6 residue with the oxadiazole $\mathrm{RAH}$ removes two amide $\mathrm{C}=\mathrm{O}$ groups and an amide $\mathrm{NH}$, connecting two hydrophobic patches that are disconnected in 5 (Figure 5, right). These findings may explain why there is an overall difference of $1.29 \mathrm{~min}$ in the retention times and 23 $\AA^{2}$ in PSA between $\mathbf{5}$ and $\mathbf{8}$ and highlight how heterocycles can be used to tune the polarities of these scaffolds.

Another important factor to consider is whether the high permeability seen for $\mathbf{8}$ is due to conformational flexibility to a more permeable conformation, or if the compound is locked into a conformation which is conducive to permeability. ${ }^{48}$ To determine this, we carried out NMR analysis in $\mathrm{CDCl}_{3}$, a nonhydrogen bonding solvent that mimics the low dielectric and lipophilic interior of the membrane. ${ }^{49}$ VT NMR analysis confirmed that the amide bonds of $\mathbf{8}$ were also non-solvent exposed in $\mathrm{CDCl}_{3}$ (Leu3, -1.0 ppb/K, Phe5, -1.3 ppb/K). NMRrestrained conformational searches showed a rigid set of conformations, all maintaining the same hydrogen bonding pattern that was seen in DMSO (Figure 6). The oxazole and oxadiazole grafts remain in a planar orientation. In fact, the only structural difference between the conformation in DMSO and chloroform is a $180^{\circ}$ flip by the proline residue. This observation indicates that heterocyclic grafts can rigidify a conformation that enables excellent permeation.
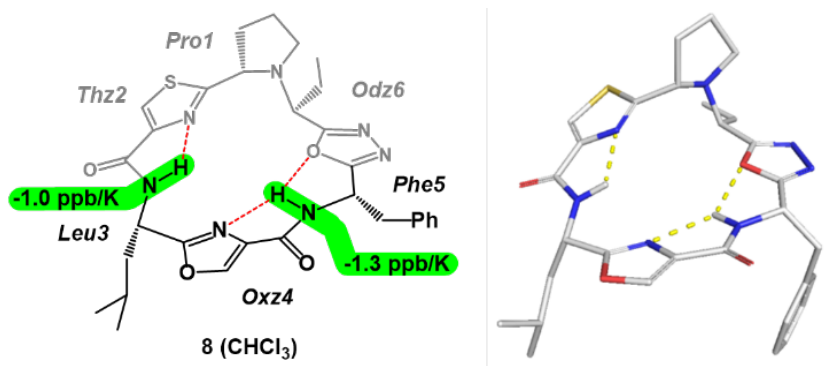

Figure 6: Lowest energy structure of 8 in chloroform determined by NMR-restrained conformational searches.

Curiously, the structure of $\mathbf{8}$ closely resembles a family of semi-peptidic natural products called Bistratamides, 18-membered macrocycles which typically contain one or more thiazole or oxazole in the backbone. Specifically, Bistratamide G features one thiazole and two oxazole grafts, and is moderately cytotoxic to the HCT-116 cancer cell line. ${ }^{50}$ Overlay of the backbone of $\mathbf{8}$ with a crystal structure of Bistratamide G shows overwhelming similarity, with expected deviations in the linker region of the oxadiazole and the proline ring (Figure 7). This comparison highlights the role of heterocycles in accessing biologically relevant conformations. Separate studies by Kihlberg and Whitty suggest that small to medium-sized macrocycles generally bind with compact, globular conformations whilst larger bRo5 macrocycles are well suited to interact with flat binding sites by edge-on or face-on approach. ${ }^{51,52}$ The planarity of $\mathbf{8}$ 
dictated by the oxazole and oxadiazole substituents suggests that azole grafted peptides can access conformational space that is more akin to larger macrocycles compared to other similarly sized macrocycles of its kind.

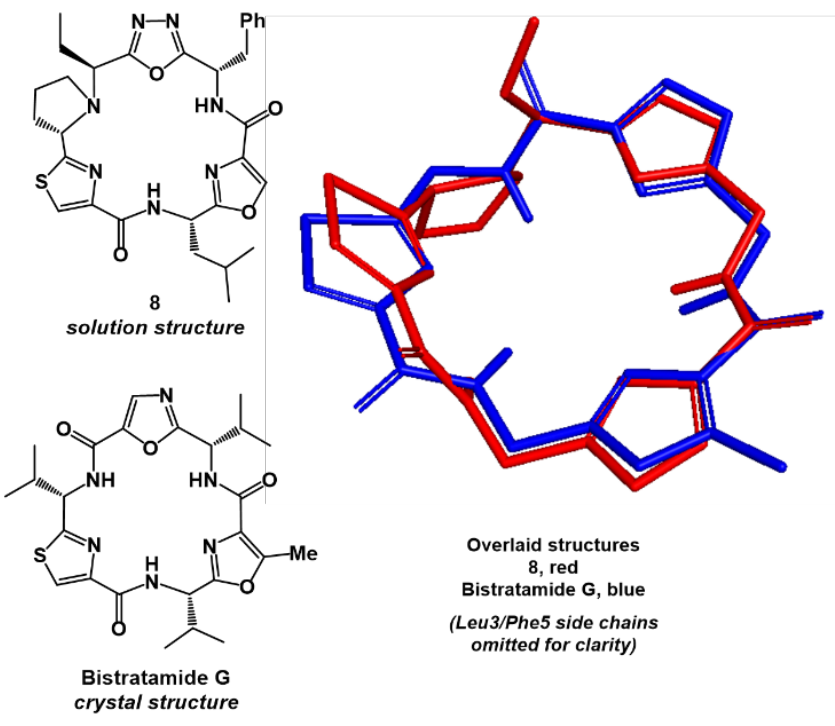

Figure 7: Overlay of the backbone of Bistratamide G (blue) with triply grafted macrocycle 8 (red).

Since the majority of the compounds synthesized do not have any classical secondary structure, we used the recently developed macrocycle conformational mapping (MCM) tool to extract further structural details derived from heterocyclic grafting. ${ }^{53}$ It is clear that the oxazole and thiazoles are important in stabilizing the structure of $\mathbf{8}$, so we produced a $\triangle \mathrm{MCM}$ map to compare the conformational relationships of $\mathbf{8}$ alongside $\mathbf{2}$, which only contains the oxadiazole RAH (Figure 8). Each of the heterocyclic grafts exhibits strong control over the $\phi / \psi$ space, occupying angles approaching $-180^{\circ}$ in $\mathbf{8}$. The conformation of the oxadiazole RAH is similar in both cases, as shown by the small magnitude of change in the vector and remains close to $-180^{\circ}$. There is a tight distribution of dihedral angles in 8 that span across very small $\phi / \psi$ space, whereas the dihedrals in $\mathbf{2}$ are spread over many regions of the MCM plot. This analysis emphasizes how heterocycles can bias dihedral angles into specific areas of macrocycle drug-like conformational space that promotes high permeability, which is of fundamental importance for the development of privileged macrocycle scaffolds based on this study. ${ }^{54}$

\section{Conclusions}

In this paper, we have initiated a research program that pursues the design and development of privileged macrocycle scaffolds with favourable permeability. This addresses a key challenge in macrocycle development by tackling the issue of permeability at an early stage. Our approach moves away from the typical amino acid-like building blocks such as $N$-methyl and D-amino acids, utilizing azole heterocycles that introduce planar conformational restraints. We have studied the structurepermeability relationships between macrocycles containing different numbers of azoles in the backbone. As more heterocycles are placed in the backbone, permeability tends to increase, although this is not always the case. The properties of the macrocycle after grafting are not always predictable, which justifies

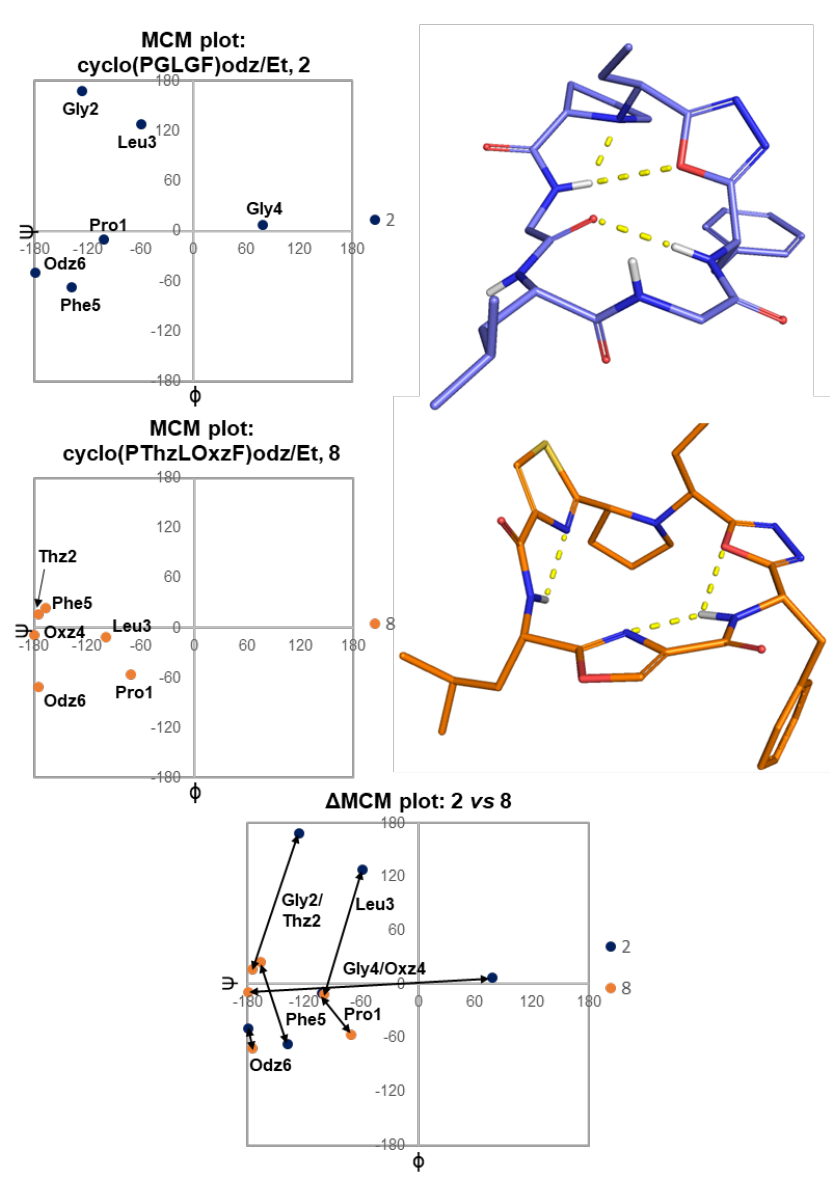

Figure 8: MCM plots of 2 and 8, and $\triangle M C M$ plot comparing 2 with 8.

the need for systematic studies in other ring sizes. The permeability landscape of azole grafted macrocycles greatly depends on their 3D conformation in solution. NMR-restrained MD simulations and conformational searches showed that a combination of oxazole and thiazole grafts arranges the backbone into a conformation where all the remaining amides are non-solvent exposed. The changes in lipophilicity of these scaffolds are tuneable as more heterocycles replace polar amide bonds. By replacing an Ala residue for the oxadiazole RAH, two hydrophobic surfaces can be connected, which leads to higher permeability and lipophilicity. ${ }^{21}$ The conformations induced by the planar heterocycles embedded in the backbone might offer a unique type of protein binding compared to other macrocycles of this size. ${ }^{51}$ Future studies will utilize heterocycles to demonstrate $\chi$ space control in addition to the $\phi / \psi$ control described here ${ }^{55,56}$ Importantly, the thiazole and oxazole motifs included in this study could conceivably be amenable to further functionalization at the C-5 position, which would extend the scope to mimic other amino acids and enable structure-activity relationships in an analogous fashion to small molecules. ${ }^{57,58}$ Our study shifts the discourse on privileged scaffolds to macrocycles and suggests permeability as the focal point, which will likely contribute to the emergence of other molecules for early stages of discovery campaigns. The discovery of privileged motifs disclosed in this work should also find use in training sets for machine learning approaches. This is because unnatural macrocycles with demonstrably high levels of passive membrane permeability represent much-needed datapoints to empower A.I.- 
driven searches for new and improved scaffolds with favourable drug-like properties.

\section{ASSOCIATED CONTENT}

Detailed experimental procedures, including synthesis; HRMS data, NMR spectra, VT-NMR and solution structure determination can be found in the supporting information. This material is available free of charge via the Internet at http://pubs.acs.org.

\section{AUTHOR INFORMATION}

\section{Corresponding Author}

*Andrei K. Yudin - Davenport Research Laboratories, Department of Chemistry, University of Toronto, Toronto, Ontario, Canada M5S 3H6; orcid.org/0000-0003-3170-9103; E-mail: andrei.yudin@utoronto.ca

\section{Author Contributions}

A.K.Y conceived the idea. G.J.S designed experiments, performed chemical synthesis, purification and characterization, computed NMR-based structures, performed unrestrained MD simulations and carried out PAMPA assays. G.J.S wrote the paper with input from A.K.Y.

\section{Funding Sources}

NSERC and Genentech are gratefully acknowledged for financial support.

\section{ACKNOWLEDGMENT}

We thank D. Burns and K. Denmans for assistance with NMR spectroscopic experiments. We also thank Prof. R. Scott Lokey, Dr Chad Townsend and Colin Kelly for assistance with PAMPA assays.

\section{ABBREVIATIONS}

DEPBT $=(3$-(diethoxyphosphoryloxy)-1,2,3-benzotriazin$4(3 \mathrm{H})$-one); DIPEA = diisopropylethyl amine, PINC $=N$-(isocyamino)triphenylphosphorane, $\mathrm{Odz}=$ Oxadiazole, $\mathrm{Oxz}=\mathrm{Ox}-$ azole, $\mathrm{Thz}=$ Thiazole, $\mathrm{RAH}=$ reduced amide heterocycle, Ro5 $=$ rule of five, $\mathrm{HBA}=$ hydrogen bond acceptor, $\mathrm{HBD}=$ hydrogen bond donor, IMHB = intramolecular hydrogen bond, PSA $=$ polar surface area.

\section{REFERENCES}

1 A. Zorzi, K. Deyle and C. Heinis, Current Opinion in Chemical Biology, 2017, 38, 24-29.

2 L. K. Buckton, M. N. Rahimi and S. R. McAlpine, Chemistry - A European Journal, 2021, 27, 1487-1513.

3 A. Henninot, J. C. Collins and J. M. Nuss, Journal of Medicinal Chemistry, 2018, 61, 1382-1414.

4 M. Egbert, A. Whitty, G. M. Keserü and S. Vajda, Journal of Medicinal Chemistry, 2019, 62, 10005-10025.

5 A. A. Vinogradov, Y. Yin and H. Suga, Journal of the American Chemical Society, 2019, 141, 4167-4181.

6 D. S. Nielsen, N. E. Shepherd, W. Xu, A. J. Lucke, M. J. Stoermer and D. P. Fairlie, Chemical Reviews, 2017, 117, 8094-8128.

7 H. Zhang and S. Chen, RSC Chemical Biology, , DOI:10.1039/D1CB00154J.

8 A. Whitty, L. A. Viarengo and M. Zhong, Organic \& Biomolecular Chemistry, 2017, 15, 7729-7735.

9 S. D. Appavoo, S. Huh, D. B. Diaz and A. K. Yudin, Chemical Reviews, 2019, 119, 9724-9752.
10 R. Jwad, D. Weissberger and L. Hunter, Chemical Reviews, 2020, 120, 9743-9789.

11 W. M. Hewitt, S. S. F. Leung, C. R. Pye, A. R. Ponkey, M. Bednarek, M. P. Jacobson and R. S. Lokey, Journal of the American Chemical Society, 2015, 137, 715-721.

12 C. L. Ahlbach, K. W. Lexa, A. T. Bockus, V. Chen, P. Crews, M. P. Jacobson and R. S. Lokey, Future Medicinal Chemistry, 2015, 7, 2121-2130.

13 A. T. Bockus, J. A. Schwochert, C. R. Pye, C. E. Townsend, V. Sok, M. A. Bednarek and R. S. Lokey, Journal of Medicinal Chemistry, 2015, 58, 7409-7418.

14 T. Rezai, B. Yu, G. L. Millhauser, M. P. Jacobson and R. S. Lokey, Journal of the American Chemical Society, 2006, 128, 2510-2511.

15 P. G. Dougherty, A. Sahni and D. Pei, Chemical Reviews, 2019, 119, 10241-10287.

16 C. K. Wang, J. E. Swedberg, P. J. Harvey, Q. Kaas and D. J. Craik, The Journal of Physical Chemistry B, 2018, 122, 2261-2276.

17 L. K. Buckton and S. R. McAlpine, Organic Letters, 2018, 20, 506-509.

18 A. Furukawa, C. E. Townsend, J. Schwochert, C. R. Pye, M. A. Bednarek and R. S. Lokey, Journal of Medicinal Chemistry, 2016, 59, 9503-9512.

19 J. Schwochert, R. Turner, M. Thang, R. F. Berkeley, A. R. Ponkey, K. M. Rodriguez, S. S. F. Leung, B. Khunte, G. Goetz, C. Limberakis, A. S. Kalgutkar, H. Eng, M. J. Shapiro, A. M. Mathiowetz, D. A. Price, S. Liras, M. P. Jacobson and R. S. Lokey, Organic Letters, 2015, 17, 2928-2931.

20 T. R. White, C. M. Renzelman, A. C. Rand, T. Rezai, C. M. McEwen, V. M. Gelev, R. A. Turner, R. G. Linington, S. S. F. Leung, A. S. Kalgutkar, J. N. Bauman, Y. Zhang, S. Liras, D. A. Price, A. M. Mathiowetz, M. P. Jacobson and R. S. Lokey, Nature Chemical Biology, 2011, 7, 810-817.

21 H. N. Hoang, T. A. Hill and D. P. Fairlie, Angewandte Chemie - International Edition, 2021, 60, 8385-8390.

22 O. Ovadia, S. Greenberg, J. Chatterjee, B. Laufer, F. Opperer, H. Kessler, C. Gilon and A. Hoffman, Molecular Pharmaceutics, 2011, 8, 479-487.

23 E. Biron, J. Chatterjee, O. Ovadia, D. Langenegger, J. Brueggen, D. Hoyer, H. A. Schmid, R. Jelinek, C. Gilon, A. Hoffman and H. Kessler, Angewandte Chemie International Edition, 2008, 47, 2595-2599.

24 C. Sohrabi, A. Foster and A. Tavassoli, Nature Reviews Chemistry, 2020, 4, 90-101.

25 Y. Goto and H. Suga, Accounts of Chemical Research, 2021, 54, 3604-3617.

26 C. J. Hipolito and H. Suga, Current Opinion in Chemical Biology, 2012, 16, 196-203.

27 T. E. McAllister, O. D. Coleman, G. Roper and A. Kawamura, Peptide Science, , DOI:10.1002/pep2.24204.

28 Y. Hosono, J. Morimoto and S. Sando, Organic \& Biomolecular Chemistry, 2021, 19, 10326-10331.

29 A. T. Frank, N. S. Farina, N. Sawwan, O. R. Wauchope, M. Qi, E. M. Brzostowska, W. Chan, F. W. Grasso, P. Haberfield and A. Greer, Molecular Diversity, 2007, 11, 115-118.

30 J. R. Frost, C. C. G. Scully and A. K. Yudin, Nature Chemistry, 2016, 8, 1105-1111.

31 S. D. Appavoo, T. Kaji, J. R. Frost, C. C. G. Scully and A. K. Yudin, Journal of the American Chemical Society, 2018, 140, 8763-8770.

32 D. S. Nielsen, H. N. Hoang, R. J. Lohman, F. Diness and D. P. Fairlie, Organic Letters, 2012, 14, 5720-5723.

33 D. S. Nielsen, H. N. Hoang, R.-J. Lohman, T. A. Hill, A. J. Lucke, D. J. Craik, D. J. Edmonds, D. A. Griffith, C. J. Rotter, R. B. Ruggeri, D. A. Price, S. Liras and D. P. Fairlie, Angewandte Chemie International Edition, 2014, 53, 12059 12063.

34 I. v. Smolyar, A. K. Yudin and V. G. Nenajdenko, Chemical Reviews, 2019, 119, 10032-10240. 
B. Diaz, S. D. Appavoo, A. F. Bogdanchikova, Y. Lebedev, T. J. McTiernan, G. dos Passos Gomes and A. K. Yudin, Nature Chemistry, 2021, 13, 218-225.

36 S. Roesner, G. J. Saunders, I. Wilkening, E. Jayawant, J. v. Geden, P. Kerby, A. M. Dixon, R. Notman and M. Shipman, Chemical Science, 2019, 10, 2465-2472.

37 C. L. Ahlbach, K. W. Lexa, A. T. Bockus, V. Chen, P. Crews, M. P. Jacobson and R. S. Lokey, Future Medicinal Chemistry, 2015, 7, 2121-2130.

38 R. E. Thompson, K. A. Jolliffe and R. J. Payne, Organic Letters, 2011, 13, 680-683.

39 S. Deng and J. Taunton, Journal of the American Chemical Society, 2002, 124, 916-917.

40 B. J. Bennion, N. A. Be, M. W. McNerney, V. Lao, E. M. Carlson, C. A. Valdez, M. A. Malfatti, H. A. Enright, T. H. Nguyen, F. C. Lightstone and T. S. Carpenter, The Journal of Physical Chemistry B, 2017, 121, 5228-5237.

41 T. J. Sereda, C. T. Mant, F. D. Sönnichsen and R. S. Hodges, Journal of Chromatography A, 1994, 676, 139-153.

42 J. M. R. Parker, D. Guo and R. S. Hodges, Biochemistry, 1986, 25, 5425-5432.

43 B. Over, P. Matsson, C. Tyrchan, P. Artursson, B. C. Doak, M. A. Foley, C. Hilgendorf, S. E. Johnston, M. D. Lee, R. J. Lewis, P. McCarren, G. Muncipinto, U. Norinder, M. W. D. Perry, J. R. Duvall and J. Kihlberg, Nature Chemical Biology, 2016, 12, 1065-1074.

44 C. K. Wang and D. J. Craik, Biopolymers, 2016, 106, 901909.

45 J. P. Snyder, Journal of the American Chemical Society, 1984, 106, 2393-2400.

46 M. Schnopp, S. Ernst and G. Haberhauer, European Journal of Organic Chemistry, 2009, 2009, 213-222.
47 T. Rezai, J. E. Bock, M. v. Zhou, C. Kalyanaraman, R. S. Lokey and M. P. Jacobson, Journal of the American Chemical Society, 2006, 128, 14073-14080.

48 V. Poongavanam, E. Danelius, S. Peintner, L. Alcaraz, G. Caron, M. D. Cummings, S. Wlodek, M. Erdelyi, P. C. D. Hawkins, G. Ermondi and J. Kihlberg, ACS Omega, 2018, 3, 11742-11757.

49 R. v. Swift and R. E. Amaro, Journal of Computer-Aided Molecular Design, 2011, 25, 1007-1017.

50 S.-L. You and J. W. Kelly, Tetrahedron, 2005, 61, 241-249.

51 E. A. Villar, D. Beglov, S. Chennamadhavuni, J. A. Porco, D. Kozakov, S. Vajda and A. Whitty, Nature Chemical Biology, 2014, 10, 723-731.

52 B. C. Doak, J. Zheng, D. Dobritzsch and J. Kihlberg, Journal of Medicinal Chemistry, 2016, 59, 2312-2327.

53 T. McTiernan, D. B. Diaz, G. J. Saunders, F. Sprang and A. $\mathrm{K}$. Yudin, RSC Chemical Biology, submitted.

54 L. A. Viarengo-Baker, L. E. Brown, A. A. Rzepiela and A. Whitty, Chemical Science, 2021, 12, 4309-4328.

55 S. M. Cowell, Y. S. Lee, J. P. Cain and V. J. Hruby, Current Medicinal Chemistry, 2004, 11, 2785-2798.

56 V. J. Hruby, G. Li, C. Haskell-Luevano and M. Shenderovich, Biopolymers, 1997, 43, 219-266.

57 N. A. Strotman, H. R. Chobanian, Y. Guo, J. He and J. E. Wilson, Organic Letters, 2010, 12, 3578-3581.

58 X.-W. Liu, J.-L. Shi, J.-X. Yan, J.-B. Wei, K. Peng, L. Dai, C.-G. Li, B.-Q. Wang and Z.-J. Shi, Organic Letters, 2013, 15, 5774-5777. 


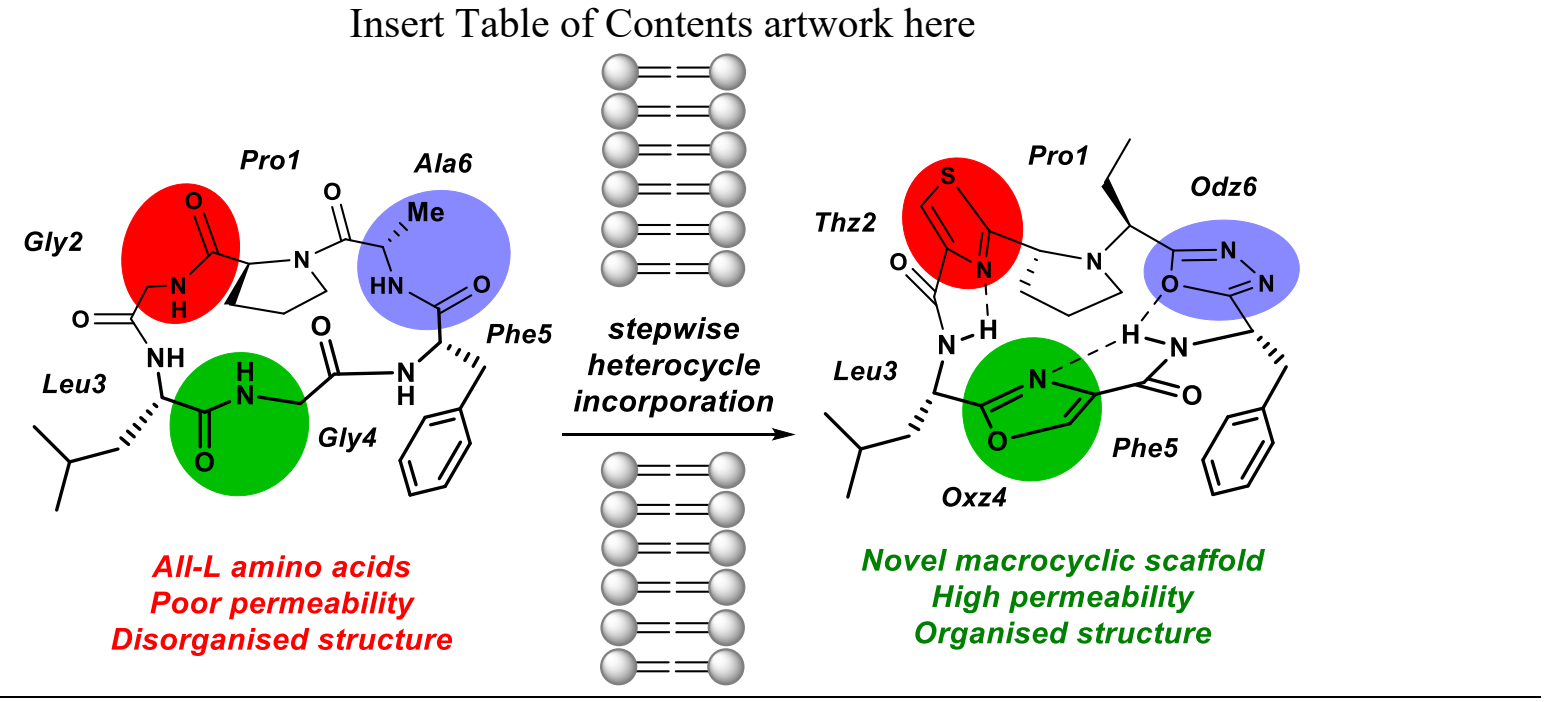

